\title{
Automatic continuity of biseparating maps
}

\author{
by \\ Jesús Araujo (Santander) and \\ KrzYsZToF JAROSz (Edwardsville, IL)
}

\begin{abstract}
We prove that a biseparating map between spaces of vector-valued continuous functions is usually automatically continuous. However, we also discuss special cases when this is not true.
\end{abstract}

1. Introduction. Assume $A, B$ are spaces of continuous functions on a set $X$ taking values in a normed space $E$. A linear map $T: A \rightarrow B$ is called separating if

$$
\|f(\cdot)\|\|g(\cdot)\| \equiv 0 \Rightarrow\|T f(\cdot)\|\|T g(\cdot)\| \equiv 0,
$$

and biseparating if $T^{-1}$ exists and is separating as well.

The concept has its source in the theory of topological lattices but is also an important generalization of multiplicative maps on Banach algebras with application to many other areas. One of such areas includes composition operators considered in ergodic theory and harmonic analysis, as any composition operator is separating. Separating and biseparating maps have been studied intensively by many authors; the reader may want to check [3, $5-9,12]$ for a large number of interesting results, examples, and applications of such maps. A recent monograph by Y. Abramovich and A. K. Kitover [2] may serve as the best first step into that area; lattice isomorphisms, Banach algebra isomorphisms, and weighted composition operators are the basic examples of separating maps, but the reader will also find there separating maps that do not belong to any of these categories. The most basic, initial questions are whether in a given setting a separating linear bijection must be biseparating, and if such a map must be automatically continuous. Such questions need typically to be answered before one can ap-

2000 Mathematics Subject Classification: Primary 47B33; Secondary 46H40, 47B38, $46 \mathrm{E} 40,46 \mathrm{E} 25$. tions.

Key words and phrases: automatic continuity, biseparating maps, vector-valued func-

Research of J. Araujo partially supported by the Spanish Dirección General de Investigación Científica y Técnica (DGICYT, PB98-1102). 
ply the theory of continuous separating maps. For example both questions have positive answers for separating linear bijections between $C(X)$ spaces with $X$ compact [11], negative in general [1], and are open in some other cases.

In this note we show that any biseparating map between spaces of bounded vector-valued functions is automatically continuous if and only if the underlying topological space $X$ has no isolated points. For maps between spaces of unbounded functions the situation is more complicated but perhaps also more interesting. If $X$ is metrizable, or connected, or locally compact, or separable, or satisfies some other simple restrictions, we again get the same condition: no isolated points. However, there are sets $X$ with no isolated points that admit biseparating maps which are discontinuous in a very strong sense. Our results are valid in both real and complex cases.

2. Preliminaries. For a completely regular space $X$ and a normed space $E$ we denote by $C(X, E)$ and $C_{\mathrm{b}}(X, E)$ the space of all continuous functions from $X$ into $E$, and the space of all bounded continuous functions from $X$ into $E$, respectively. If $E$ is equal to the scalar field, we write $C(X)$ in place of $C(X, E)$. We equip $C_{\mathrm{b}}(X, E)$ with the topology of uniform convergence on $X$, and $C(X, E)$ with the topology of uniform convergence on compact subsets of $X$. For normed spaces $E, F$ we denote by $L^{-1}(E, F)$ the set of all linear bijections from $E$ onto $F$.

For a function $f \in C(X)$ it may be often convenient to extend $f$ to a continuous function on $\beta X$, the maximal compactification of $X$. In general, for $x \in \beta X \backslash X$, the value of $f(x)$ may be infinite. There are, however, completely regular spaces $X$ with a point $x_{0} \in \beta X \backslash X$ such that the value of $f\left(x_{0}\right)$ is finite for all $f \in C(X)$; the set of all points in $\beta X$ with this property is called the realcompactification of $X$ and is denoted by $r X$. Since we have $C(X)=C(r X)$, the natural domain for a continuous function on $X$ is $r X$, not $X$. Hence we will often consider realcompactifications, or alternatively, we will assume that the completely regular spaces under consideration are realcompact. All compact sets are clearly realcompact, also all subsets of Euclidean spaces are realcompact, or even more generally all metrizable spaces of nonmeasurable cardinality are realcompact ([10, p. 232]).

We will often refer to the following result from [4].

TheORem 1. Suppose that $X$ and $Y$ are realcompact spaces, E, $F$ are normed spaces, and either

- $T$ is a linear biseparating map from $C(X, E)$ onto $C(Y, F)$, or

- $E, F$ are infinite-dimensional and $T$ is a linear biseparating map from $C_{\mathrm{b}}(X, E)$ onto $C_{\mathrm{b}}(Y, F)$. 
Then there exists a surjective homeomorphism $h: Y \rightarrow X$ and a map $J: Y \rightarrow L^{-1}(E, F)$ such that $(T f)(y)=(J(y))(f(h(y)))$ for every $f$ in the domain of $T$ and $y \in Y$.

Notice the result can also be applied if $E, F$ are finite-dimensional and $T$ is defined on $C_{\mathrm{b}}(X, E)$ since in that case we have $C_{\mathrm{b}}(X, E) \simeq C_{\mathrm{b}}(\beta X, E)=$ $C(\beta X, E)$. Based on the above theorem, each time we are asked about continuity of a biseparating map $T$, we can compose the map with $f \mapsto f \circ h^{-1}$, and assume without loss of generality that $X=Y$ and that

$$
(T f)(x)=(J(x))(f(x)) \quad \text { for every } f \text { and } x \in X .
$$

Hence, if the normed spaces $E, F$ are finite-dimensional any biseparating map $T$ must be automatically continuous. On the other hand, if $X$ contains an isolated point $x_{0}$ and $E$ is infinite-dimensional then one can trivially define a discontinuous biseparating map on $C(X, E)$ or $C_{\mathrm{b}}(X, E)$ : take any discontinuous linear bijection $J_{0}$ from $E$ onto itself and put

$$
(T f)(x)= \begin{cases}f(x) & \text { if } x \neq x_{0} \\ J_{0}\left(f\left(x_{0}\right)\right) & \text { if } x=x_{0} .\end{cases}
$$

In the next section we show that, except for some very special sets $X$, this is basically the only way one can get a discontinuous biseparating map.

Recall that a point $x_{0}$ in a topological space is called a $P$-point if for each sequence $\left(U_{n}\right)$ of neighborhoods of $x_{0}$, the intersection $\bigcap_{n=1}^{\infty} U_{n}$ contains again a neighborhood of $x_{0}$; if a topological space is first countable, for example if it is metrizable, then the only $P$-points are the isolated points. A completely regular topological space is called a $P$-space if each point of the space is a $P$-point. Among the metric spaces, the $P$-spaces coincide with the discrete spaces. We will need the following properties of $P$-spaces ([10, p. 63]).

Proposition 1. For a completely regular space $X$ the following conditions are equivalent:

- $X$ is a P-space,

- any real-valued continuous function on $X$ is locally constant,

- any $G_{\delta}$ subset of $X$ is open.

\section{Results}

Theorem 2. Assume $X, Y$ are realcompact spaces, $E, F$ are normed spaces, and $T: C_{\mathrm{b}}(X, E) \rightarrow C_{\mathrm{b}}(Y, F)$ is a linear biseparating map. If $Y$ has no isolated points then $T$ is continuous.

Notice that for a finite-dimensional space $E$ the space $C_{\mathrm{b}}(X, E)$ can be identified with $C(\beta X, E)$, where $\beta X$ is the maximal compactification of $X$. Hence for finite-dimensional spaces $E, F$ our theorem follows immediately 
from Theorem 1; we do not even need the assumption that $Y$ has no isolated points.

Proof. By Theorem 1 we may assume without loss of generality that $Y=X$ and that $T$ is of the form (2.1). Suppose that $T$ is not continuous and let $\left(f_{n}\right)_{n=1}^{\infty}$ be a sequence of elements in $C_{\mathrm{b}}(X, E)$ such that

$$
\left\|f_{n}\right\|<1 / n^{2} \text { and }\left\|T f_{n}\right\|>n \quad \text { for } n \in \mathbb{N} .
$$

Let $x_{1} \in X$ be such that $\left\|\left(T f_{1}\right)\left(x_{1}\right)\right\| \geq 1$. Since $x_{1}$ is not isolated, there exists an $x_{2} \neq x_{1}$ with $\left\|\left(T f_{2}\right)\left(x_{2}\right)\right\| \geq 2$. Continuing this process, we can inductively construct a sequence $\left(x_{n}\right)_{n=1}^{\infty}$ of different points in $X$ such that

$$
\left\|\left(T f_{n}\right)\left(x_{n}\right)\right\| \geq n \quad \text { for } n \in \mathbb{N} .
$$

It is easy to see that it is possible to get a subsequence $\left(x_{n_{k}}\right)$ of $\left(x_{n}\right)$ with the property that for each $k_{0} \in \mathbb{N}, x_{n_{k_{0}}}$ does not belong to the closure of $\left\{x_{n_{k}}: k \neq k_{0}\right\}$. Without loss of generality we suppose that $\left(x_{n_{k}}\right)=\left(x_{n}\right)$. Let $\left(U_{n}\right)_{n=1}^{\infty}$ be a sequence of open subsets of $X$ and $\left(g_{n}\right)$ a sequence of continuous bounded real-valued functions on $X$ such that for any $n \in \mathbb{N}$,

$$
x_{n} \in U_{n}, \quad g_{n}\left(x_{n}\right)=1=\left\|g_{n}\right\| \quad \text { and } \quad \operatorname{supp} g_{n} \subset U_{n} .
$$

Since $\left\|g_{n} f_{n}\right\| \leq\left\|f_{n}\right\|<1 / n^{2}$ we can define a function $f$ in $C_{\mathrm{b}}(X, E)$ by

$$
f:=\sum_{j \in \mathbb{N}} g_{j} f_{j}
$$

From (2.1) and since $g_{n}\left(x_{n}\right)=1$ and $g_{j}\left(x_{n}\right)=0$ for $j \geq n$ we get

$$
\begin{aligned}
\left\|(T f)\left(x_{n}\right)\right\| & =\left\|\left(J\left(x_{n}\right)\right)\left(f\left(x_{n}\right)\right)\right\|=\left\|\left(J\left(x_{n}\right)\right)\left(\sum_{j \in \mathbb{N}} g_{j}\left(x_{n}\right) f_{j}\left(x_{n}\right)\right)\right\| \\
& =\left\|\left(J\left(x_{n}\right)\right)\left(f_{n}\left(x_{n}\right)\right)\right\|=\left\|\left(T\left(f_{n}\right)\right)\left(x_{n}\right)\right\| \geq n .
\end{aligned}
$$

Since our space contains only bounded functions, the contradiction shows continuity of $T$.

Theorem 3. Assume $X, Y$ are realcompact spaces and $E, F$ are both infinite-dimensional normed spaces. Then

(i) if the interior of the set of $P$-points of $Y$ is empty then every linear biseparating map $T: C(X, E) \rightarrow C(Y, F)$ is continuous,

(ii) if the interior of the set of $P$-points of $Y$ is not empty then there is a discontinuous linear biseparating map $T: C(Y, F) \rightarrow C(Y, F)$,

(iii) if $\operatorname{dim} E=\operatorname{dim} F$ then all points of $Y$ are $P$-points if and only if for any linear bijection $J$ from $E$ onto $F$ (continuous or not) the map $T(f):=J \circ f$ is well defined and biseparating from $C(Y, E)$ onto $C(Y, F)$.

Corollary 1. For realcompact spaces $X, Y$ and normed spaces $E, F$ there is a linear biseparating map between $C(X, E)$ and $C(Y, F)$ if and only if $X$ and $Y$ are homeomorphic and either 
- $E$ and $F$ are isomorphic, or

- $\operatorname{dim} E=\operatorname{dim} F$ and $Y$ is a P-space.

REMARK 1. There is another useful way to think about these results. Notice that if $J$ is a linear bijection from a normed space $E$ onto itself, then we can phrase the usual definition of continuity of $J$ as follows:

$J$ is continuous iff the transformation $f \mapsto J(f)$ maps $C\left(\mathbb{N}^{*}, E\right)$ into itself,

where $\mathbb{N}^{*}$ denotes the one-point compactification of the set of natural numbers. Now, based on our results we can replace the set $\mathbb{N}^{*}$ in the definition above with a set $X$ if and only if $X$ is not a $P$-space.

Proof of Theorem 3. (i) Assume that the interior of the set of $P$-points of $Y$ is empty and $T: C(X, E) \rightarrow C(Y, F)$ is a biseparating map. As before by Theorem 1 we may assume that $Y=X$ and that $T$ is of the form (2.1).

First we are going to prove that $J(y): E \rightarrow F$ is continuous for every $y \in X$. Assume that there is a $y_{0} \in X$ such that $J\left(y_{0}\right)$ is discontinuous. If $y_{0}$ is not a $P$-point we put $x_{0}:=y_{0}$. If $y_{0}$ is a $P$-point we define $V_{n}=$ $\{x \in X:\|J(x)\|>n\}$. Since the map $\|\cdot\| \circ J: X \rightarrow[0, \infty]$ sending each $y \in X$ into $\|J(y)\|$ is continuous, all of the sets $V_{n}$ are neighborhoods of $y_{0}$, and so is $\bigcap_{n=1}^{\infty} V_{n}$. Moreover for any $x \in \bigcap_{n=1}^{\infty} V_{n}$ the map $J(x)$ is discontinuous. Since the interior of the set of $P$-points is empty, the set $\bigcap_{n=1}^{\infty} V_{n}$ contains a point which is not a $P$-point. We denote by $x_{0}$ one of such points.

Let $\left(e_{n}\right)$ be a sequence in $E$ such that

$$
\left\|e_{n}\right\|<1 / n^{2} \text { and }\left\|J\left(x_{0}\right)\left(e_{n}\right)\right\|>n \text { for } n \in \mathbb{N} \text {. }
$$

Let $\mathbf{e}_{n}$ be the constant function equal to $e_{n}$. Since $x_{0}$ is not a $P$-point there is a sequence of open neighborhoods $U_{n}$ of $x_{0}$ with

$$
U_{n} \subset\left\{x \in X:\left\|\left(T \mathbf{e}_{n}\right)(x)\right\|=\left\|J(x)\left(e_{n}\right)\right\|>n\right\}
$$

and such that the intersection of all $U_{n}$ is not a neighborhood of $x_{0}$; we may also assume that $\bar{U}_{n+1} \subset U_{n}$ for $n \in \mathbb{N}$. It follows that $x_{0}$ belongs to the closure of the following set:

$$
A:=\bigcup_{n=0}^{\infty}\left(U_{n+1}-U_{n+2}\right) .
$$

For each $n \in \mathbb{N}$, let $g_{n} \in C_{\mathrm{b}}(X)$ be such that

$$
\left\|g_{n}\right\|=1=g_{n}(x) \quad \text { for } x \in U_{n+1}, \quad \text { and } \quad \operatorname{supp} g_{n} \subset U_{n} \text {. }
$$

Put

$$
g:=\sum_{n=1}^{\infty}\left(g_{n}-g_{n+2}\right) e_{n+1}
$$


since $\left\|g_{n}-g_{n+2}\right\| \leq 2$ and $\left\|e_{n}\right\|<1 / n^{2}$ the series is convergent and $g \in$ $C(X, E)$. For any $k \in \mathbb{N}$ and any $x_{k+1} \in U_{k+1}-U_{k+2}$ we have

$$
\left(g_{n}-g_{n+2}\right)\left(x_{k+1}\right)= \begin{cases}1-1=0 & \text { if } n<k, \\ 1-0=1 & \text { if } n=k, \\ 0-0=0 & \text { if } n>k\end{cases}
$$

Hence

$$
\begin{aligned}
\left\|(T g)\left(x_{k+1}\right)\right\| & =\left\|\left(J\left(x_{k+1}\right)\right)\left(\sum_{n=1}^{\infty}\left(g_{n}-g_{n+2}\right)\left(x_{k+1}\right) e_{n+1}\right)\right\| \\
& =\left\|\left(J\left(x_{k+1}\right)\right)\left(e_{k+1}\right)\right\|=\left\|\left(T \mathbf{e}_{k+1}\right)\left(x_{k+1}\right)\right\|>k+1 .
\end{aligned}
$$

Since $x_{0}$ belongs to the closure of $\bigcup_{n=0}^{\infty}\left(U_{n+1}-U_{n+2}\right)$ we get $\left\|(T g)\left(x_{0}\right)\right\|$ $=\infty$, and this contradiction proves the continuity of $J(y)$ for every $y \in X$.

Suppose now that $T$ is not continuous. Then there is a compact subset $K$ of $X$ and a sequence $\left(f_{n}\right)$ in $C(X, E)$ with $p_{K}\left(f_{n}\right)<1 / n^{2}$ and $p_{K}\left(T f_{n}\right)>n$.

Let $y_{n} \in K$ be such that $\left\|\left(T f_{n}\right)\left(y_{n}\right)\right\|>n$ for each $n \in \mathbb{N}$. Notice that the set $\left\{y_{n} \in K: n \in \mathbb{N}\right\}$ is infinite since otherwise there would be a point $y_{n_{0}} \in K$ with $\left\|\left(T f_{n}\right)\left(y_{n_{0}}\right)\right\|>n$ for infinitely many $n \in \mathbb{N}$, contrary to the continuity of $J\left(y_{n_{0}}\right)$. We may assume that $y_{n} \neq y_{m}$ when $n \neq m$, and take a sequence $\left(U_{n}\right)_{n=1}^{\infty}$ of pairwise disjoint open subsets of $X$ such that $y_{n} \in U_{n} \subset\left\{x \in X:\left\|f_{n}(x)\right\|<1 / n^{2}\right\}$ for $n \in \mathbb{N}$. Let $\left(g_{n}\right)_{n=1}^{\infty}$ be a sequence in $C_{\mathrm{b}}(X)$ such that $\operatorname{supp} g_{n} \subset U_{n}$ and $g_{n}\left(y_{n}\right)=1$ for $n \in \mathbb{N}$. It is easy to see that

$$
f:=\sum_{k=1}^{\infty} g_{k} f_{k} \in C(X, E)
$$

while, by $(2.1),\left\|(T f)\left(y_{n}\right)\right\|=\left\|\left(T f_{n}\right)\left(y_{n}\right)\right\|>n$ so $T f$ is not bounded on $K$. Since this is not possible we conclude that $T$ must be continuous.

(ii) Assume now that the interior $\mathcal{I}$ of the set of $P$-points of $Y$ is not empty and let $h_{0}$ be a continuous real-valued function on $Y$ such that $h_{0}(x)=1$ for $x \in X \backslash \mathcal{I}$, and $h_{0}\left(z_{0}\right)=0$ at some point $z_{0} \in \mathcal{I}$. Put $\mathcal{A}=h_{0}^{-1}(0)$. By Proposition $1, \mathcal{A}$ is an open subset of $\mathcal{I}$, and consequently of $X$; clearly, being a zero set, $\mathcal{A}$ is also closed. Let $J$ be a discontinuous linear bijection from $F$ onto itself and define $T: C(Y, F) \rightarrow C(Y, F)$ by

$$
(T f)(x)= \begin{cases}f(x) & \text { if } x \notin \mathcal{A}, \\ J(f(x)) & \text { if } x \in \mathcal{A} .\end{cases}
$$

The map $T$ is obviously biseparating but we need to check that it is well defined, that is, that $T f$ is continuous for any continuous $f$. This is of course true on the clopen subset $X \backslash \mathcal{A}$. Let $f \in C(Y, F)$ and let $z \in \mathcal{A}$. By Proposition 1 the function

$$
\mathcal{A} \ni x \mapsto\|f(x)-f(z)\|
$$


is constant on a neighborhood of $z$, hence $f$, and so $T f$, are both locally constant and consequently continuous.

(iii) As above we assume without loss of generality that $X=Y$ and that $h$ is the identity map. Suppose that $\operatorname{dim} E=\operatorname{dim} F$ and that there is a point $x_{0} \in X$ which is not a $P$-point. We will see that there exists a linear bijection $J$ from $E$ onto $F$ such that the map $T(f):=J \circ f$ is not well defined.

Since $J$ is not continuous, there exists a sequence $\left(e_{n}\right)$ of norm-one elements of $E$ such that $\left\|J e_{n}\right\|>n^{3}$ for $n \in \mathbb{N}$. On the other hand, since $x_{0}$ is not a $P$-point, there exists a sequence $\left(U_{n}\right)_{n=1}^{\infty}$ of neighborhoods of $x_{0}$ such that the intersection of all $U_{n}$ is not a neighborhood of $x_{0}$. We may assume that $\bar{U}_{n+1} \subset U_{n}$ for $n \in \mathbb{N}$. Consequently, $x_{0}$ belongs to the closure of at least one of the following sets:

$$
A_{1}:=\bigcup_{n=1}^{\infty}\left(U_{2 n}-U_{2 n+2}\right) \quad \text { or } \quad A_{2}:=\bigcup_{n=1}^{\infty}\left(U_{2 n-1}-U_{2 n+1}\right) .
$$

Without loss of generality we may assume that $x_{0} \in \bar{A}_{2}$.

For each $n \in \mathbb{N}$, let $g_{n} \in C_{\mathrm{b}}(X), 0 \leq g_{n} \leq 1$, be such that

$$
g_{n}\left(U_{2 n-1}-U_{2 n+1}\right)=1 \text { and } \operatorname{supp} g_{n} \subset U_{2 n-2}-U_{2 n+2} .
$$

It is clear that the map

$$
f:=\sum_{k=1}^{\infty} \frac{1}{k^{2}} g_{k} e_{k}
$$

belongs to $C(X, E)$. Also, according to the description of $T$ and to the fact that $x_{0} \in \bar{A}_{2}$, we deduce that $\left\|(T f)\left(x_{0}\right)\right\|=\infty$, which is absurd. Consequently, $X$ is a $P$-space.

To prove the other implication we just repeat the above argument in (ii) with $\mathcal{A}=X$.

To prove the Corollary, notice that if $Y$ is not a $P$-space, then it contains a point $y_{0}$ which is not a $P$-point, and consequently, as in the proof of Theorem 3(i), $J\left(y_{0}\right)$ is continuous (and bijective). On the other hand, by Theorem 1, $\left(T^{-1} g\right)(x)=(K x)\left(g\left(h^{-1}(x)\right)\right)$ for some $K \in L^{-1}(F, E)$. In particular $K(h(y))$ is the inverse of $J(y)$ (see [4] for the proof of Theorem 1), which implies in particular that $h(y)$ is not a $P$-point and, consequently, $E$ and $F$ are isomorphic.

The $P$-sets without isolated points are rather unusual, they cannot be locally compact or metrizable. An example of such a set can be found in [10], below we provide another one.

EXAMPLE 1. Let $\Omega$ be an uncountable set and put $X=\{0,1\}^{\Omega}$. We say that a function $f$ on $X$ depends on countably many coordinates if there is a 
countable subset $\Omega_{0}$ of $\Omega$ such that

$$
f(x)=f\left(x^{\prime}\right) \quad \text { whenever } x(\omega)=x^{\prime}(\omega) \text { for all } \omega \in \Omega_{0} .
$$

Notice that $f$ depends on countably many coordinates if and only if $f=$ $f_{0} \circ p_{\Omega_{0}}$ where $\Omega_{0}$ is a countable subset of $\Omega, p_{\Omega_{0}}$ is the natural projection from $\{0,1\}^{\Omega}$ onto $\{0,1\}^{\Omega_{0}}$ and $f_{0}$ is a function on $\{0,1\}^{\Omega_{0}}$. We denote by $C_{\aleph_{0}}(X)$ and $C_{\aleph_{0}}^{\mathrm{b}}(X)$ the set of all scalar-valued functions that depend on countably many variables, and bounded functions with that property, respectively. We introduce on $X$ the coarsest topology such that all functions in $C_{\aleph_{0}}(X)$ are continuous. That topology $\tau$ of $X$ has a base that can be described as follows:

$$
\left\{p_{\Omega_{0}}^{-1}(A): A \subset\{0,1\}^{\Omega_{0}}, \operatorname{card}\left(\Omega_{0}\right) \leq \aleph_{0}\right\} .
$$

Since the uniform limit of a sequence of functions depending on countably many coordinates each also has this property, $C_{\aleph_{0}}^{\mathrm{b}}(X)$ is a self-adjoint uniform algebra so $X$ can be viewed as a subset of the maximal ideal space of that algebra. Since any subset of a compact Hausdorff space is completely regular, so is $X$.

Suppose that $f$ is a continuous real-valued function on $X$ and fix $x_{0} \in X$. Without loss of generality, we assume that $x_{0}(\omega)=0$ for $\omega \in \Gamma \subset \Omega$, and $x_{0}(\omega)=1$ for $\omega \in \Omega \backslash \Gamma$. Since, for any $n \in \mathbb{N}$, the set $U_{n}=\{x \in X$ : $\left.\left|f(x)-f\left(x_{0}\right)\right|<1 / n\right\}$ is open, it contains an open set of the form

$$
V_{n}=\left\{x \in X: x\left(\Omega_{1, n}\right) \subset\{0\}, x\left(\Omega_{2, n}\right) \subset\{1\}\right\},
$$

for some countable sets $\Omega_{1, n} \subset \Gamma, \Omega_{2, n} \subset \Omega \backslash \Gamma$. Put $\Omega_{1}=\bigcup_{n=1}^{\infty} \Omega_{1, n}$, $\Omega_{2}=\bigcup_{n=1}^{\infty} \Omega_{2, n}$; clearly $\Omega_{1}, \Omega_{2}$ are countable and disjoint. Now

$$
U_{0}=\left\{x \in X: x\left(\Omega_{1}\right) \subset\{0\}, x\left(\Omega_{2}\right) \subset\{1\}\right\}
$$

is a neighborhood of $x_{0}$ and $f$ is constant on $U_{0}$.

Since the operation of taking realcompactification preserves the class of $P$-sets $([10$, Ex. 8A.5]) as well as the class of spaces without isolated points, $r X$ is an example of a realcompact $P$-set without isolated points. The authors do not know if the set $X$ is already realcompact for each uncountable set $\Omega$.

\section{References}

[1] Y. A. Abramovich and A. K. Kitover, A solution to a problem on invertible disjointness preserving operators, Proc. Amer. Math. Soc. 126 (1998), 1501-1505.

[2] —, - Inverses of disjointness preserving operators, Mem. Amer. Math. Soc. 679 (2000).

[3] J. Araujo, Separating maps and linear isometries between some spaces of continuous functions, J. Math. Anal. Appl. 226 (1998), 23-39. 
[4] J. Araujo, Realcompactness and Banach-Stone theorems, submitted; available at http://www.matesco.unican.es/ araujo/isom3.dvi and at http://xxx.lanl.gov/pdf/ math/0010292.

[5] J. Araujo, E. Beckenstein and L. Narici, When is a separating map biseparating?, Arch. Math. (Basel) 67 (1996), 395-407.

[6] - , - - Biseparating maps and homeomorphic realcompactifications, J. Math. Anal. Appl. 192 (1995), 258-265.

[7] J. Araujo and K. Jarosz, Separating maps on spaces of continuous functions, in: Proc. 3rd Conf. on Function Spaces, Contemp. Math. 232, Amer. Math. Soc., 1999, $33-38$.

[8] H. G. Dales, Banach Algebras and Automatic Continuity, London Math. Soc. Monographs 24, Oxford Univ. Press, New York, 2000.

[9] J. J. Font and S. Hernández, On separating maps between locally compact spaces, Arch. Math. (Basel) 63 (1994), 158-165.

[10] L. Gillman and M. Jerison, Rings of Continuous Functions, Van Nostrand, 1960.

[11] K. Jarosz, Automatic continuity of separating linear isomorphisms, Canad. Math. Bull. 33 (1990), 139-144.

[12] J.-S. Jeang and N.-C. Wong, Weighted composition operators of $C_{0}(X)$ 's, J. Math. Anal. Appl. 201 (1996), 981-993.

Departamento de Matemáticas,

Estadística y Computación

Facultad de Ciencias

Universidad de Cantabria

Avda. de los Castros, s. n.

E-39071 Santander, Spain

E-mail: araujoj@unican.es

http://www.matesco.unican.es/〜araujo/
Department of Mathematics and Statistics Southern Illinois University Edwardsville, IL 62026, U.S.A. E-mail: kjarosz@siue.edu http://www.siue.edu/ ${ }^{\sim}$ jarosz/

Received July 6, 2001

Revised version November 13, 2002 